\title{
Remarks on Generalized Contractions
}

\author{
D. Göhde
}

Abstract. The main result concerns an extension of Schauder's fixed point theorem to not necessarily continuous maps $T$ which satisfy

$$
\|T x-T y\| \leq a\|x-y\|+b \max \{\|T x-x\|,\|T y-y\|\} \quad \text { with } a \geq 0, b \in(0,1) .
$$

Keywords. Fixed points, generalized contractions, nonexpansive mappings

Mathematics Subject Classification (2000). Primary 47H10, secondary 54H25

\section{Introduction}

Since the seventies of last century, such maps in Banach or metric spaces are investigated which are, sometimes, called generalized contractions, nonexpansive type mappings, or the like. In one of the first papers in this field, Roux and Soardi [11] proved the existence of a unique fixed point of a self-map of a bounded, closed and convex set $C$ in a uniformly convex Banach space, with the property

$$
\|T x-T y\| \leq \alpha\|x-y\|+\beta(\|x-T x\|+\|y-T y\|),
$$

where $\alpha, \beta$ are positive functions, restricted by the condition $\alpha+2 \beta=1$. Thus (1) may be interpreted as a convex combination of the conditions on nonexpansive $(\beta=0)$, and Kannan maps $(\alpha=0$; see, e.g., [8, 9]). Goebel, Kirk, and Shimi [5] extended this result to maps obeying

$$
\|T x-T y\| \leq \alpha\|x-y\|+\beta(\|x-T x\|+\|y-T y\|)+\gamma(\|x-T x\|+\|y-T y\|),
$$

$\alpha, \beta, \gamma$ being nonnegative constants, with $\alpha+2 \beta+2 \gamma=1$. Here $C$ need not be bounded, but $T$ has to be continuous - which is not given by (1) or (2) itself.

Without any conditions on the Banach spaces and the map T, except for (1), but $\alpha+2 \beta=1$, in 1980 Greguš [6] proved the existence of a fixed point of $T: C \mapsto C$ in a closed convex set $C$.

Dietrich Göhde: Heinrich-Budde-Str. 19, D-04157 Leipzig, Germany 
Also, correspondig mappings in complete metric spaces $(M,|\cdot, \cdot|)$ have been already investigated (e.g., in [2, 7]; see also the survey in [10]). As a typical example we mention the condition imposed on a selfmap $T$ of $M$ (see[3]):

$$
\begin{aligned}
|T x, T y| \leq & \alpha \max \left\{|x, y|,|x, T x|,|y, T y|, \frac{1}{2}(|x, T y|+|y, T x|)\right\} \\
& +\beta \max \{|x, T x|,|y, T y|\}+\gamma(|x, T y|+|y, T x|)
\end{aligned}
$$

with $\alpha \geq 0, \beta>0, \gamma>0$, and $\alpha+\beta+2 \gamma=1$.

The unusually looking term $|x, T y|+|y, T x|$ turns out to be essential in order to prove inf $|x, T x|=0$ here and in other papers (e.g., in [1] where multivalued maps are considered too). But on condition that the space has a convexity structure it had been possible to dispense with this term. Thus, e.g., in Ćirić's paper [4] the existence of a fixed point could be proved if $T: M \mapsto M$ merely fulfils

$$
|T x, T y| \leq a|x, y|+b \max \{|x, T x|,|y, T y|\}
$$

with $a, b>0$, and $a+b=1$, extending Greguš's result.

It seems that in all papers on the topic in question the coefficients within the conditions on the mappings must obey limitations as $\alpha+2 \beta(+2 \gamma) \leq 1$, or $a+b \leq 1$. On the other hand, some proofs (e.g., in [6]) make one conjecture these limitations could be relaxed a little. In the present paper, this expectation can, indeed, be verified, but it will also be seen that a more remarkable releasing, probably, asks for additional assumptions: here compactness will be imposed.

\section{Results}

In 2.1, we first will provide a basically known fact in appropriate form for use in the sequel. In 2.2 , the possibility of relaxing the condition on the coefficients will in principle be confirmed in case of (1). The existence of a fixed point in case of the Banach space version of $(3)$, with $a \geq 0, b \in(0,1)$, will be proved in 2.3 for a selfmap $T$ of a compact convex set.

2.1. Let $T$ be a selfmap of a complete metric space $(M,|\cdot, \cdot|)$ which satisfies $(3)$ with nonnegative constants $a, b$. Using the triangle inequality and (3),

$$
|x, y| \leq|x, T x|+|T x, T y|+|T y, y| \leq a|x, y|+(1+b)(|x, T x|+|y, T y|),
$$

therefore, if $a<1$,

$$
|x, y| \leq \frac{1+b}{1-a}(|x, T x|+|y, T y|)
$$

Again, by the triangle inequality,

$$
|y, T y| \leq|y, x|+|x, T x|+|T x, T y| \leq(1+a)|x, y|+(1+b)|x, T x|+b|y, T y|,
$$


and, if $b<1$,

$$
|y, T y| \leq \frac{1+a}{1-b}|x, y|+\frac{1+b}{1-b}|x, T x| .
$$

From this, it follows

Lemma. Let $T$ satisfy (3) with $a, b \in[0,1)$. Then $T$ has a fixed point iff $\inf _{x \in M}|x, T x|=0$. The fixed point $x$ is unique, and $T$ is continuous at $x$.

Proof. Let $M_{n}=\left\{x \in M:|x, T x|<\frac{1}{n}\right\}$. If $\inf |x, T x|=0, M_{n} \neq \emptyset$ for all integers $n>0$. Choose $x_{n} \in M_{n}$. As, by (4), the diameters of $M_{n}$ tend to zero, $\left\{x_{n}\right\}$ is a Cauchy sequence. For its limit $y$ and for $x=x_{n}$, (5) tells that $|y, T y|=0$.

Uniqueness of $y$ is an immediate consequence of (3) or (4). Using (5) for a sequence $\left\{y_{n}\right\}$ converging to a fixed point $x=T x$, we get $\lim \left|y_{n}, T y_{n}\right|=0$, and therefore $\lim \left|T x, T y_{n}\right|=0$ by (3).

2.2. In order to confirm in principle the possibility of weakening the condition (1) with respect to the coefficients, we will prove the following

Proposition. Let $C \neq \emptyset$ be a closed convex subset of a Banach space, and let $T: C \mapsto C$ be a map which satisfies (1) for $x, y \in C$, with constants $\alpha \in$ $(0,1), \beta \in\left(0, \frac{1}{2}\right)$. Then there exists a positive smooth function $\delta(\alpha), \alpha \in(0,1)$, such that $T$ has a fixed point if $\alpha+2 \beta<1+\delta(\alpha)$.

Proof. For the most part, we will follow the procedure of Greguš [6]. Let $x_{n}:=$ $T^{n} x_{0}, n \geq 0$, for some $x_{0} \in C$. Then, by (1),

$$
\left\|x_{n+1}-x_{n}\right\| \leq q^{n}\left\|x_{1}-x_{0}\right\|, \quad q=\frac{\alpha+\beta}{1-\beta} .
$$

The case $q=1$ is equivalent to $\alpha+2 \beta=1$; we will consider $q \geq 1$. The crucial consequence of (1) is

$$
\begin{aligned}
\left\|x_{3}-x_{1}\right\| & \leq \alpha\left\|x_{2}-x_{0}\right\|+\beta\left(\left\|x_{1}-x_{0}\right\|+\left\|x_{3}-x_{2}\right\|\right) \\
& \leq\left[\alpha(1+q)+\beta\left(1+q^{2}\right)\right]\left\|x_{1}-x_{0}\right\| \\
& =q(q+1-2 \beta)\left\|x_{1}-x_{0}\right\|
\end{aligned}
$$

using (6) and $\alpha=(1-\beta) q-\beta$.

Now we estimate the displacement of $z:=\frac{1}{2}\left(x_{2}+x_{3}\right)$ by $T$ :

$$
\|T z-z\| \leq \frac{1}{2}\left(\left\|T z-x_{2}\right\|+\left\|T z-x_{3}\right\|\right) .
$$

By (1) it follows

$$
\left\|T z-x_{2}\right\| \leq \alpha\left\|z-x_{1}\right\|+\beta\left\|x_{2}-x_{1}\right\|+\beta\|T z-z\| .
$$


As

$$
\left\|z-x_{1}\right\| \leq \frac{1}{2}\left(\left\|x_{2}-x_{1}\right\|+\left\|x_{3}-x_{1}\right\|\right) \leq \frac{1}{2}[q+q(q+1-2 \beta)]\left\|x_{1}-x_{0}\right\|
$$

and

$$
\begin{aligned}
\left\|T z-x_{2}\right\| & \leq\left[\frac{\alpha}{2} q(q+2-2 \beta q)+\beta q\right]\left\|x_{1}-x_{0}\right\|+\beta\|T z-z\| \\
\left\|T z-x_{3}\right\| & \leq \frac{\alpha}{2}\left\|x_{3}-x_{2}\right\|+\beta\left\|x_{3}-x_{2}\right\|+\beta\|T z-z\| \\
& \leq\left(\frac{\alpha}{2}+\beta\right) q^{2}\left\|x_{1}-x_{0}\right\|+\beta\|T z-z\|
\end{aligned}
$$

we get

$$
(1-\beta)\|T z-z\| \leq \frac{1}{2} q[(\alpha+\beta) q+\alpha+\beta-\alpha \beta]\left\|x_{1}-x_{0}\right\|
$$

Taking into account $\frac{(\alpha+\beta)}{(1-\beta)}=q, \frac{\beta}{(1-\beta)}=\frac{(q-\alpha)}{(1+\alpha)}$, we get

$$
\|T z-z\| \leq Q(q, \alpha)\left\|x_{1}-x_{0}\right\|, \quad \text { with } \quad Q(q, \alpha)=\frac{1}{2} q\left[q^{2}+\frac{q+\alpha^{2}}{1+\alpha}\right] .
$$

Now, if $Q(q, \alpha)<1$, then $\inf _{x \in C}\|T x-x\|=0$, and $T$ has a fixed point according to the lemma $(a=\alpha, b=2 \beta)$. Otherwise, if we had $d:=\inf \|T x-x\|>0$, we could find $x_{0}$ with $\left\|x_{1}-x_{0}\right\|<d Q^{-1}$, and therefore by (8), $\|T z-z\|<d$.

Because of $\frac{\partial Q(q, \alpha)}{\partial q}>0$, and $Q(1, \alpha)=1-\frac{\alpha(1-\alpha)}{2(1+\alpha)}<1$, the equation $Q(\cdot, \alpha)=1$ has a unique solution $q^{*}=q^{*}(\alpha)>1$ which is smooth in $(0,1)$, and $Q(q, \alpha)<1$ for $q<q^{*}(\alpha)$. Finally, as $1-\beta=\frac{(1+\alpha)}{(1+q)}, \alpha+2 \beta=1+\alpha+\beta-(1-\beta)=1+(1+\alpha) \frac{q-1}{q+1}$ is an increasing function of $q$; therefore

$$
\alpha+2 \beta<1+\delta(\alpha), \quad \text { with } \delta(\alpha):=(1+\alpha) \frac{q^{*}(\alpha)-1}{q^{*}(\alpha)+1}
$$

is equivalent to $q<q^{*}(\alpha)$.

Remark. As indicated already, this is a result on principle, and of minor numerical significance: e.g., $\delta(0,5) \approx 0,026$. Also taking $z=\lambda x_{2}+(1-\lambda) x_{3}$ with $\lambda$ chosen optimal (instead of $\lambda=\frac{1}{2}$ above), $\delta$ could be enlarged but marginally. In case of the more general condition corresponding to (3) the procedure used here will yield a $\delta$ even smaller.

2.3. In order to improve the result of 2.2 substantially we impose compactness. So we are able to derive a specific extension of Schauder's theorem. 
Theorem. Let $K \neq \emptyset$ be a compact convex subset of a Banach space, and $T: K \mapsto K$ a map satisfying, for $x, y \in K$,

$$
\|T x-T y\| \leq a\|x-y\|+b \max \{\|T x-x\|,\|T y-y\|\}
$$

with constants $a, b: a \geq 0,0<b<1$. Then $T$ has a fixed point.

Notice that $T$ does not need to be continuous.

Proof. We have to show that $\|T x-x\|$ has infimum 0 in $K$.

Suppose $d:=\inf _{x \in K}\|T x-x\|>0$. Let $\left\{y_{1}, \ldots, y_{n}\right\}$ be an $\varepsilon$-net in $K$ such that the balls $B\left(y_{k}, \varepsilon\right)=\left\{x \in K:\left\|x-y_{k}\right\|<\varepsilon\right\}, k=1, \ldots, n$, are covering $K$; $\varepsilon$ will be determined later. Define $\eta_{k}^{0}(x)=\max \left\{\varepsilon-\left\|x-y_{k}\right\|, 0\right\}$. Then

$$
\eta_{k}(x):=\frac{\eta_{k}^{0}(x)}{\sum_{i=1}^{n} \eta_{k}^{0}(x)}, \quad k=1, \ldots, n
$$

make up a partition of unity in $K$, the support of $\eta_{k}$ being $\overline{B\left(y_{k}, \varepsilon\right)}$.

Owing to convexity,

$$
S x:=\sum_{k=1}^{n} \eta_{k}(x) T y_{k}
$$

is a continuous selfmap of $K$. Consider, e.g., $x \in B\left(y_{1}, \eta\right)$, and assume that $y_{1}, \ldots, y_{m}$ are all net-points within $B\left(y_{1}, 2 \varepsilon\right)$. Then $S x$ is a convex combination of $T y_{1}, \ldots, T y_{m}$.

Set $T y_{i}-y_{i}=v_{i}$, and let $\left\|v_{l}\right\|=\max \left\{v_{1}\|, \ldots,\| v_{m} \|\right\}$. There is a continuous linear functional $f$ of norm one, and $f\left(v_{l}\right)=\left\|v_{l}\right\| \geq d$. For $k \in\{1, \ldots, m\} \backslash\{l\}$,

$$
\left\|T y_{l}-T y_{k}\right\| \geq\left\|v_{l}-v_{k}\right\|-\left\|y_{l}-y_{k}\right\|>\left\|v_{l}-v_{k}\right\|-4 \varepsilon
$$

and, by (9), $\left\|T y_{l}-T y_{k}\right\| \leq a\left\|y_{l}-y_{k}\right\|+b\left\|v_{l}\right\|$, thus $\left\|v_{l}-v_{k}\right\|<4(1+a) \varepsilon+b\left\|v_{l}\right\|$. Hence

$$
f\left(v_{l}\right)-\operatorname{Re} f\left(v_{k}\right)=\operatorname{Re} f\left(v_{l}-v_{k}\right) \leq\left\|v_{l}-v_{k}\right\|<b\left\|v_{l}\right\|+4(1+a) \varepsilon
$$

whence $\operatorname{Re} f\left(v_{k}\right)>(1-b)\left\|v_{l}\right\|-4(1+a) \varepsilon$.

Finally, for $x \in B\left(y_{1}, \varepsilon\right)$, and $k=1, \ldots, m$,

$$
\operatorname{Re} f\left(T y_{k}-x\right) \geq \operatorname{Re} f\left(v_{k}\right)-\left\|y_{k}-x\right\|>(1-b)\left\|v_{l}\right\|-(7+4 a) \varepsilon \geq \frac{1-b}{2} d
$$

if $\varepsilon=\varepsilon_{0}:=\frac{(1-b) d}{2(7+4 a)}$. Because $S x$ is a convex combination of the $T y_{k}$, we have $\operatorname{Re} f(S x-x)>0$ for $x \in B\left(y_{1}, \varepsilon_{0}\right)$ and likewise for $x \in B\left(y_{i}, \varepsilon_{0}\right), i=2, \ldots, n$. Therefore, $S x \neq x$ in all of $K$ which contradicts Schauder's fixed point theorem, and thus $d>0$ is false: necessarily $d=0$. 
Thanks to compactness, for proving the existence of a fixed point it will suffice to use, instead of the lemma, only inequality (5) in 2.1. This inequality is valid even for all $a \geq 0$. Indeed, select a sequence $\left\{x_{n}\right\}$ converging to a point $y \in K$, with $\lim \left\|T x_{n}-x_{n}\right\|=0$ : then (5), for this $y$, and $x=x_{n}$, will entail $\|T y-y\|=0$.

\section{References}

[1] Chandra, M., Mishra, S. N., Singh, S. L., and Rhoades, B. E., Coincidences and fixed points of nonexpansive type multi-valued and single-valued maps. Indian J. Pure Appl. Math. 26 (1995)(5), 393 - 401.

[2] Ćirić, Lj. B., Generalized contractions and fixed point theorems. Publ. Inst. Math. (Beorgrad) 12 (1971), 19 - 26.

[3] Ćirić, Lj. B., On some nonexpansive type mappings and fixed points. Indian J. Pure Appl. Math. 24 (1993), 145 - 149.

[4] Ćirić, Lj. B., On a generalization of a Greguš fixed point theorem. Czechoslovak. Math. J. 50 (2000), $449-458$.

[5] Goebel, K., Kirk, W. A., and Shimi, T. N., A fixed point theorem in uniformly convex spaces. Boll. Un. Mat. Ital. 7 (1973)(4), $67-75$.

[6] Greguš, M., A fixed point theorem in Banach spaces. Boll. Un. Mat. Ital. A (5) 17 (1980), $193-198$.

[7] Hardy, G. E., and Rogers, T. D., A generalization of a fixed point theorem of Reich. Canad. Math. Bull. 16 (1973), 201 - 206.

[8] Kannan, R., Some results on fixed points II. Amer. Math. Monthly 76 (1969), $405-408$.

[9] Reich, S., Remarks on fixed points. Atti Accad. Naz. Lincei Rend. Cl. Sci. Fis. Mat. Natur. 52 (1972)(8), $689-697$.

[10] Rhoades, B. E., A comparison of various definitions of contractive mappings. Trans. Amer. Math. Soc. 226 (1977), $257-290$.

[11] Roux, D., and Soardi, P., Alcune generalizzazioni del Teorema di BrowderGöhde-Kirk (in Italian). Atti. Accad. Naz. Lincei Rend. Cl. Sci. Fis. Mat. Natur. 52 (1972)(8), $682-688$.

Received June 14, 2006 